\title{
Development of an ethics education curriculum for critical care trainees in Canada: from knowledge synthesis to bedside application
}

\author{
Joshua T. Landry ${ }^{1} \cdot$ Sabira Valiani ${ }^{2}$. \\ Thomas C. Foreman ${ }^{3,4} \cdot$ Rakesh V. Patel ${ }^{5,6}$
}

Published online: 24 November 2015

(C) Springer International Publishing AG 2015

\begin{abstract}
Intensivists require a specialized approach to learning and managing complex bioethical issues due to the immediacy, uncertainty and gravity of medical decisions made when caring for the critically ill. A systematic examination of the literature and solicited feedback from experienced Intensivists has demonstrated that the knowledge and skill required to understand and navigate complex bioethical dilemmas is not often taught with the rigor necessary for independent clinical practice. This prompted us to design a bioethics curriculum for adult critical care medicine trainees to fill a significant void in ethics knowledge and reasoning within critical care training. The curriculum consists of six self-learning, online, case-based modules, and interactive group discussions. It is to be integrated into an existing Critical Care Academic Half Day schedule and completed over a 1-2 year period. This paper presents a detailed explication of the problem, and puts forward our developed solution - a comprehensive bioethics curriculum.
\end{abstract}

Keywords Ethics $\cdot$ Critical care $\cdot$ Curriculum $\cdot$ Medical trainee $\cdot$ Education

Joshua T. Landry

joslandry@toh.on.ca

1 The Champlain Centre for Health Care Ethics, The Ottawa Hospital, 1053 Carling Ave, Ottawa, ON K1Y 4E9, Canada

2 PGY4, Adult Critical Care Medicine, University of Ottawa The Ottawa Hospital, 1053 Carling Ave, Ottawa, ON K1Y 4E9, Canada

3 Department of Clinical and Organizational Ethics, The Ottawa Hospital, Ottawa, Canada

4 Department of Medicine, University of Ottawa. The Ottawa Hospital, 1053 Carling Ave, Ottawa, ON K1Y 4E9, Canada

5 Department of Medicine, University of Ottawa, Ottawa, Canada

6 Department of Critical Care, The Ottawa Hospital, 1053 Carling Ave, Ottawa, ON K1Y 4E9, Canada 


\section{Introduction}

Graduate medical education, which may include residency and fellowship training, is necessary for the consolidation and refinement of a physician's knowledge, skills and reasoning within their base or subspecialty. It is a journey of practical, hands-on education and training. In Canada, it incorporates non-medical expert roles of: communicator, collaborator, leader, scholar, professional and advocate. These should provide some foundational exposure to the recognition, analysis and navigation of bioethical problems. Unfortunately, the knowledge and skill required to manage complex bioethical dilemmas is frequently not taught with the intent of converting and incorporating principles of bioethics into practical, everyday bedside application. (Stevens et al. 2002) Specialists in intensive care medicine (Intensivists) and critical care trainees do not typically think of bioethics principles as tools analogous to a stethoscope or bedside ultrasound. This prompted us to design a curriculum to fill a void in bioethical knowledge and reasoning in adult critical care medicine training. The goal of this paper is to present a detailed explication of this problem, and put forward our solution - a comprehensive bioethics curriculum to be presented in six structured sessions to adult critical care medicine trainees. Although our curriculum has been designed specifically for critical care medicine, it has been structured to be useful across specialties with minimal modification.

Intensivists have a specialized need for applied ethics. The rate at which ethical dilemmas may arise in critical care may be more immediate, and less foreseeable than in other areas of medicine. The critical care setting is fraught with the twin hazards of time pressure and clinical uncertainty that overarches the complexity and severity of critical illness. In the ICU, clinicians commonly have a limited amount of time within which challenging management decisions have to be made. Moreover, these decisions must be taken, frequently, during emotionally charged situations for patients that Intensivists may hardly know at all. Increasing the complexity of decision making in this setting is the uncertainty of diagnosis and/or prognosis and of the benefit of sophisticated treatments for any given individual patient. Finally, the emotionally problematic and draining toll of end-of-life decision-making (that often arise precipitously) on patients, families, substitute decision-makers and health-care professionals alike, in this setting, all conspire to make ethical dilemmas much more compressed and stressful than other clinical environments. For Intensivists to successfully practice ethical medicine in this environment, appropriately focused education and training is necessary to aid recognition and subsequent generation of a plan to work through ethical dilemmas in ways that traditional evidence based, or physiological or systemsoriented medicine may not allow.

\section{Literature review}

\section{Current training}

The current situation in the North American context demonstrates a paucity of literature discussing the pedagogical methods used to aid medical trainees in general, and critical care residents more specifically, in developing the required acumen and skill to 
appropriately deal with ethical dilemmas. (Kane et al. 1998) What is more troubling is that this is likely due to an insufficient focus and lack of curricula that address ethical issues during the training of physicians. Stevens and colleagues report that, in residency training before intensive care unit rotations, $78.8 \%$ of residents responded that they received 'none' or 'too little' formal teaching sessions on ethical principles; $69.3 \%$ responded that they received 'none' or 'too little' case-based, patient centered ethics education; and $69.2 \%$ responded that they received 'none' or 'too little' informal discussion about ethics. They also indicated that the amount of ethics education during an intensive care unit rotation often fared worse, where $71.0 \%$ of residents reported that they had formal teaching sessions on ethical principles in 'none' or 'too little' of their training; $77.4 \%$ responded that they had 'none' or 'too little' case-based, patient centered ethics education; $87.1 \%$ responded that they had 'none' or 'too little' time spent predicting survival in ICU patients; and $87.1 \%$ responded had 'none' or 'too little' time spent breaking bad news, as well as leading discussions about end-of-life decisions. (Stevens et al. 2002, 291-2) These statistics demonstrate that the current bioethics education infrastructure for medical trainees is insufficient (at least in Ontario medical schools which made up $71.2 \%$ of surveyed residents), and that a more responsive ethics curriculum is not only desirable, but crucial.

\section{The ethical issues}

ICU physicians commonly face difficult ethical challenges that may include issues of consent and capacity, and substitute decision-making. Intensive care units provide care for patients with a myriad of conditions and illnesses, and frequently, patients lack capacity and are unable to make their own medical decisions. Consequently, it may not be possible for consent to be obtained directly from the patient and providers may need to rely on previously expressed wishes and consent from a substitute decision-maker to proceed with an intervention. Interpreting advance directives or verbally-expressed previous wishes can be challenging on its own, but the difficulties of determining how to proceed with a treatment or treatment plan may be compounded when substitute decision-makers are involved who are unfamiliar with what the patient would have wanted, or even impose their own values on the decision-making process.

Other significant ethical challenges to North American ICUs involve appropriately managing end-of-life care. Specifically, ethical issues involving withholding and withdrawing of life-sustaining interventions are of particular interest since it is often difficult for providers to determine when aggressive treatments may no longer be appropriate, and a change to palliative care indicated. (Heaney et al. 2007; Mani 2003; Moore et al. 2008; Reynolds et al. 2005) This difficulty may in part be due to simply having high-technology medical interventions available. The advances in medical knowledge and technology have allowed providers to keep the most critically-ill patients alive in cases where, historically, they would have died. One of the great challenges to our use of medicine over the last century might be understood as the can vs. should problem (a formulation of David Hume's 'is-ought' problem); that is, just because we can use particular aggressive interventions to save dying patients, does not mean that we should. (Hume 1739)

Lastly, consider the notion of futility. What are often considered "futile treatments" may be particularly difficult to address since there is no commonly agreed upon 
definition of the term, and the more frequently proposed versions (i.e., quantitative, qualitative, or physiological) have been highly criticized due to their limited applicability. Despite this, the literature reveals that North American health-care providers commonly opine that they are guilty of providing what they perceive as futile or probably futile treatments, with much of the literature documenting the frequency and cost of these treatments coming from the ICU. The ICU has been specifically studied because of the high monetary cost associated with providing care in this setting. Huynh and colleagues, for example, report from a study of ICU physicians in Canada that "as many as $87 \%$ [of physicians] believed that futile treatment had been provided in their ICU in the past year," and in Europe " $27 \%$ of ICU clinicians believed that they provided 'inappropriate' care to at least 1 patient." (Huynh et al. 2013, 1888) From their investigation of five ICUs in California, they found that $232(20.6 \%)$ patients, over a 3-month period, had received futile or probably futile treatment, as indicated by treating physicians.

These are troubling statistics since it is estimated that $47.1 \%$ of hospital deaths in the United States, and approximately $50 \%$ of hospital deaths in Canada, involved ICU care. (Niederman and Berger 2010; Palda et al. 2005) In a different study of Canadian ICUs, it was found that over the previous year futile treatments had been provided to a majority of patients, with physicians estimating that $87 \%$ of patients had received such treatments, and nurses estimating that proportion to be $95 \%$. Furthermore, $27 \%$ of physicians and $34 \%$ of nurses believed that, at the time of responding to the study's survey, some of their current patients were receiving futile treatment. (Palda et al. 2005) The aforementioned challenges and dilemmas of daily ICU practice suggest that it is unrealistic to expect that Intensivists could easily, reasonably and confidently understand and/or navigate them without appropriate skills. Therein, we ventured to design a bioethics curriculum to address some of these needs.

\section{Addressing the problem}

\section{Recommended content}

Although the foundational level of ethical knowledge and skill is low among critical care trainees, Downar and colleagues suggest that this fact is easily remedied by further education. In particular, a desire for further knowledge in conflict resolution and substitute decision-making was expressed to improve trainee confidence and proficiency. (Downar et al. 2012) A partial overlap is seen in the findings of Kane and colleagues, who note that the content of formal ethics curricula currently in place in the U.S. most often addresses withdrawing life support, advance directives, clinical investigation, and emerging therapies. This type of ethics education is sparse, however, as only $17 \%$ of survey respondents indicated that they had such a curriculum in place. (Kane et al. 1998) Other topics perceived necessary for critical care trainee education were: teaching about bioethical principles; improving communication skills - including those needed to lead end-of-life discussions, break bad news, and resolve conflict; the duty to treat; futility and determining death; ethics consultation; and rights of children and the cognitively impaired. (Crausman and Armstrong. 1996; Mularski et al. 2001; Stevens et al. 2002; Van Mook et al. 2009) 


\section{Recommended delivery}

How a critical care curriculum ought to be delivered to trainees was agreed upon fairly consistently across the literature. In the existing curricula, Kane and colleagues (1998) found that ethics education was commonly delivered by several different methods: case conferences ( $77 \%$ of cases), speaker series (77\% of cases), bibliographies for independent reading (38\% of cases), research (19\% of cases), and graduate courses in bioethics (15\% of cases). More specifically, it is recommended that critical care education be delivered by interactive, practical, case-based approaches that teach at the bedside when possible. Teaching within the clinical context has a number of advantages. According to Crausman and Armstrong, learning in this environment

dispels the erroneous notion that biomedical ethics is something other than an integrated part of medical practice. It keeps the patient and family involved as active participants. It allows for brief analysis of and recognition that many daily medical decisions are in fact ethically based. Finally, it encourages true integration of the medical, social, and ethical dimensions of the biopsychosocial model. $(1996,79)$

When bedside teaching is not possible, or a more formal approach is sought, casebased methods have been demonstrated as highly effective forms of adult teaching and learning for physicians. (Ahern et al. 2012; Crausman and Armstrong 1996) Additionally, standardized patient or family exercises have demonstrated their effectiveness in improving ethical knowledge for critical care trainees. It appears that although a formal didactic method may be necessary to present the foundations of an ethics curriculum, trainee involvement in actual clinical activities, or at least, simulated activities, would be required for maximal success.

For the delivery of such a curriculum, it is recommended that an interdisciplinary approach be taken where clinical ethicists lead, at very least, the formal foundational education sessions. Intensivists who guide critical care trainees day-to-day, generally speaking, are not "universally well versed in ethical principles, well trained in end-oflife decision-making, or adept at listening, eliciting preferences, and communicating about these issues in current time-pressured clinical environments." (Stevens et al. 2002, 294-295) However, they could most effectively demonstrate their expertise through the exercise and integration of ethical skills at the bedside or through experiential case discussions.

\section{Our approach}

In response to a determined gap in bioethics education (Stevens et al. 2002), we created a bioethics curriculum for critical care trainees that encompasses essential content and effective adult learning methods to maximize learning. Specifically, our curriculum will be integrated into the existing Adult Critical Care Medicine Academic half-day schedule at the University of Ottawa Hospital. It will be completed over a 2-year period, and facilitated by a clinical ethicist. This ethicist will lead six case-based sessions, each addressing common, yet troublesome ethical issues found in critical care medicine. The 
ethicist shall work in collaboration with an Intensivist who is willing to support the bedside education and navigation of challenging ethical issues.

Prior to each session, trainees have access to a learning case, online supplementary resources, a pre-test, and the opportunity to submit their own cases for discussion. Group session teaching will align with recommendations in the existing literature whereby they will consist of a short didactic foundational component delivered by an ethicist, who will then lead case-based discussions with trainees to facilitate practical knowledge synthesis \& application. (Stevens et al. 2002) The topics chosen for each of the six sessions also align with recommendations made for content in the existing literature (Crausman and Armstrong. 1996; Mularski et al. 2001; Stevens et al. 2002; Van Mook et al. 2009), and reflect the nature and significance of the subject matter that a typical patient will experience through their critical illness. While the subject matter has been specified to the context of critical care medicine in Ontario, Canada, the curriculum has been presented in a standardized way such that it has the ability to be utilized within academic health science centers elsewhere, and for different medical/ surgical specialities. Final evaluation of the curriculum will be through a short answer post-test that is similar to the pre-test, as well as a brief verbal case presentation for which the trainee will discuss their approach for resolution. We will use these presentations to help develop a competency assessment tool. Trainees will be encouraged to provide anonymous feedback about the curriculum at its completion, so that it may be iteratively improved to meet learning needs. Data collection will consist of the short answer pre- and post-test scores, module pre-test scores, case presentation evaluation, and trainee feedback.

Additionally, the curriculum has been developed to satisfy the "CanMEDS" framework, as required by the Royal College of Physicians and Surgeons of Canada. This framework has been designed to articulate particular knowledge, skills, and abilities that specialist physicians need to achieve optimal patient outcomes, and "is based on the seven roles that all physicians need to have, to be better doctors: Medical Expert, Communicator, Collaborator, Manager, Health Advocate, Scholar, and Professional." (Royal College of Physicians and Surgeons of Canada 2005) A brief introduction to each of the six curriculum topics is presented below.

\section{Session 1: ethical reasoning and decision-making - identifying and working through difficult ethical dilemmas}

This first session begins by challenging the trainees to think about what ethical dilemmas are, how they arise, and in what ways ethical decision-making is valuable in critical care medicine. This topic will fulfil a demonstrated gap in critical care training as presented in the existing literature. (Stevens et al. 2002; Miles et al. 1989) The discussion progresses to identifying and analyzing common ethical principles, and engages the trainee to utilize these principles in their recognition of ethical issues specific to their critically ill patients. Finally, this session will conclude by briefly reviewing two frameworks for addressing ethical issues. It will first present the IDEA framework which has been developed to assist trainees in identifying and addressing clinical ethics issues. Next, the Accountability for Reasonableness (A4R) framework will be examined to aid trainees in recognizing and addressing organizational ethics issues. (Thompson et al. 2006; Trillium Health 2015) 
Suggestions for further discussion post-presentation include: exploring ethically challenging cases trainees have encountered, and how they might approach them differently post-session, how to approach complexities in addressing ethical issues at the core of a dilemma, and ensuring that trainees are aware of the ethics resources available at their current institution. This session should help partially fulfill the CanMEDS role requirements for: Professional, Health Advocate, and Medical Expert.

\section{Session 2: rights, responsibilities, and interests - understanding the health care consent Act in critical care medicine}

This session focuses on an issue frequently responsible for ethical concern in the critical care setting: patient capacity and consent to treatment. This topic was specifically chosen to inform issues frequently arising in the author's experience, documented in the literature, and addressed in the legal system. (Straus and Stelfox 2007; Cuthbertson v. Rasouli) The session is specific to the Ontario context, but offers many foundational insights into dealing with this perplexing topic. First, the trainees are presented with a case dealing with patient incapacity and substitute decision-making. Next, the definition and requirements of capacity are discussed. Here the trainees will review "capacity" as understood in Ontario's Health Care Consent Act (HCCA), and be provided with a helpful tool for assisting in the assessment of capacity in the clinical setting. (The "Aid to Capacity Evaluation" tool) (Etchells 2015) Patient incapacity is explored with particular attention to substitute decision-making, and the determination of best interests. The distinction between a power of attorney and substitute decision-maker is highlighted in this section, as well as other pertinent sections of the HCCA including substitute decision-maker role/responsibility, and elements of informed consent.

Further discussion may be directed toward current methods trainees use to determine capacity, difficulties they have in doing so, and the importance of documenting all conversations surrounding this demanding topic. Additionally discussing the complexities that may arise when patients who lack capacity disagree with the decisions that their SDM may make on their behalf, and the importance, to some extent, of respecting those disagreements may be fruitful. This session should help partially fulfill the CanMEDS role requirements for: Professional, Communicator, and Medical Expert.

\section{Session 3: developing a plan of care - ethical considerations}

The main focus of this session is to explore the ethical complexities and challenges of developing a plan of care in the critical care setting. This session begins by evaluating the specifics of pressures unique to the ICU, and is recommended to consider key information that may be helpful in developing a plan of care, such as identifying: who should be participating in decision making, what the medical facts are, where the patient's goals and values lie and whether a trial of intensive care may be appropriate. (Miles et al. 1989, 705) This information is obtained by utilizing a particular tool to determine: whether there are any ethical issues that need to be addressed, and what treatment or treatments may be ethically appropriate for a particular patient. The tool used in this session is known as the IDEA framework and, while introduced previously, 
its exploration is the main focus of this session. While presenters may wish to use a different framework of their choosing, the IDEA framework has been chosen for use in this curriculum because of its support in the literature, and effectiveness in the clinical setting at the authors' home institution. (The Ottawa Hospital) Further points to enhance or stimulate discussion may include how the aforementioned framework might be integrated into everyday practice, given the trainee's previous experience. One might ask whether there are have been any previous ethical concerns in developing a plan of care that would certainly have been helped by implementing this framework, or, if there are situations in which a more specialized ethics consultation would still have been needed, and why.

This session will assist trainees in their critical thinking, and should help partially fulfill the CanMEDS role requirements for: Professional, Communicator, Collaborator, and Manager.

\section{Session 4: when the plan of care must change - navigating ethical decision-making and communication with families}

This session is meant to allow learners to develop approaches to changing the plan of care to comfort care, and familiarize them with ethical issues surrounding this difficult task; a component noted in the existing literature as particularly challenging. (Miles et al. 1989; Heaney et al. 2007; Mani 2003; Moore et al. 2008; Reynolds et al. 2005) The ethical distinction between withdrawing and withholding care is first addressed. Next, a time frame for withdrawing care is presented, and strategies are reviewed to change the goals of care. Frequent family meetings are emphasized. This session is presented with literature specific to the ICU, and with time frames often experienced in the critical care setting. Finally, the concept of futility is introduced. This is a nuanced concept that may be extremely value laden and hinge on the definition of "benefit" to the individual patient. Suggestions for further discussion post-presentation should focus on communication strategies. For example, include a discussion of strategies that may help ease the burden on families changing the goals of care to comfort, or how to frame palliative care in an ICU context. Additional discussion points include how to discuss the concept of medical futility with families in a sensitive manner, and dealing with extreme viewpoints or demands. For example, even if an Intensivist does not offer a therapy, the family may still demand it, and communication strategies to deal with this would be very useful.

This fourth session will encourage trainees to identify ethical issues surrounding withholding and withdrawing care, and changing goals of care, and should help partially fulfill the CanMEDS role requirements for: Communicator, Collaborator, Professional, and Manager.

\section{Session 5: End-of-life care and dying in the ICU}

To address a component that has been noted in the existing literature as particularly challenging, this session is designed to focus on palliative care and the dying process. (Miles et al. 1989; Heaney et al. 2007; Mani 2003; Moore et al. 2008; Reynolds et al. 2005) The Intensivist, once palliation is agreed upon as a treatment plan, should strive 
to achieve the best balance of symptom control, based on medical indications and the patient's values.

We then move on to discuss organ donation and definitions of death. The learners are asked to present definitions of death that they may have come across in clinical practice from a religious, spiritual, or cultural standpoint. We emphasize that the dying process and end-of-life care must be completely and explicitly separate in clinical process in order to avoid any potential conflict of interest. Of course, however, our definition of death (brain death, cardiac death) has largely been developed in light of the ability to transplant organs, and provide longevity for organ recipients. Suggestions for further discussion post-presentation include review of the questions included in the case study. As well, learners could be asked to discuss the pros and cons of each organ procurement strategy, based on ethical principles and from a practical standpoint. Alternatively, a short session or discussion could also be facilitated by the organ donation team, in order to better understand how families are approached for organ donation, and the ethical framework in which the organ donation team works.

This fifth session is particularly comprehensive and should help partially fulfill the CanMEDS role requirements for: Medical Expert, Collaborator, Communicator, Health Advocate, and Professional.

\section{Session 6: (special topics) euthanasia, physician assisted death, and the right to Die}

The final session's purpose is to provide trainees with ethical guidance on special topics. We have chosen to focus on the difficult challenges that arise in jurisdictions where patients are granted a right to die, and the perceived permissibility of euthanasia and physician-assisted death. As Landry, Foreman, and Kekewich note elsewhere, the ethical issues that arise relating to this topic are of paramount importance in Canada, as the country is currently in the process of creating legislation to regulate assisted death. (2015) The requirement to legislate has come after the Supreme Court of Canada deemed provisions in the Criminal Code prohibiting the practice to be unconstitutional. Other topics may be chosen to correspond with particular issues at an organization (e.g., issues particular to pediatric intensive care, or those related to end-of-life decision making where policies are not clear), or to address broader bioethical issues affecting patients across the country.

This session begins by encouraging trainees to clarify the utilized terminology by exploring Canadian Medical Association definitions of "Euthanasia" and "Physician Assisted Suicide/Death." It is noted that while the definitions are helpful, confusion may arise depending on one's interpretation of active, versus passive euthanasia. Next, we discuss national, professional and organizational guiding positions on the topic, and further encourage the trainees to think critically about some of the ethical questions that may arise when discussing euthanasia and physician assisted death. Lastly this session presents several common arguments for and against the permissibility of euthanasia and physician assisted death. Further discussion may be stimulated by encouraging the trainees to think about whether they have heard about or witness any cases in which euthanasia or physician assisted death may have been appropriate, or even requested by a patient. What happened? Why might it have been appropriate or inappropriate to provide such treatments? How did the patient's or physicians views shape the conversations that were had? 
This session deals with important ethical issues that will likely affect Intensivists in the not-so-distant future. It encourages thoughtful reflection on the possible benefits and harms of euthanasia and physician assisted death, and should help partially fulfill the CanMEDS role requirements for: Professional, Communicator, and Medical Expert.

\section{Discussion}

This curriculum has the potential to be suitably trainee responsive for many reasons. First, it follows a typical patient's course through an ICU, thus providing a relevant timeframe where common ethical dilemmas originate. Our hope is that by facilitating a better understanding of these dilemmas, and using salient teaching methods, trainees will be better equipped and more confident at navigating and resolving ethical dilemmas. Second, while the type and frequency of ethical dilemmas may have regional variation, our curriculum addresses the fundamental ethical issues experienced clinically. It can be easily amended to suit standards across Canadian provinces and for non-Canadian jurisdictions. Third, our curriculum also has the potential to serve as a guideline to develop or form other ethics curricula for other specialties, including but not limited to geriatric or cancer care ethics, or pediatrics. Lastly, the curriculum has included an evaluation tool to measure the learners' improvement from pre- to post-curriculum.

The formal didactic method with integrated and interactive case-based learning provides an optimal adult learning structure for trainees to appreciate the foundational theoretical concepts, work through those concepts with other learners, share experiences and insecurities and apply these concepts to actual clinical cases. Practical aspects of ethics are heavily emphasized in this course: from the initial approach and recognition of a bioethics dilemma, to capacity assessment, to practical aspects of end-of-life care.

To assure the quality of our curriculum content and delivery methods, we asked the Director of Education from the Faculty of Medicine's Office of Continuing Professional Development, at the University of Ottawa, to review our curriculum. From a professional development perspective, the proposed course structure was sound, the tone was professional, and the content was well organized and clear to achieve the stated objectives. Additionally, this work has been endorsed by the University of Ottawa, Faculty of Medicine, with an Educational Initiatives in Residence Education Grant post completion.

While there are obvious strengths to this curriculum, there may be areas for improvement. Although we believe that practical and interactive case-based learning is the best format for the presentation of a bioethics, one challenge is that critical care trainees may not gain a full appreciation for extremely nuanced topics, such as futility and the euthanasia debate, through this format. A bibliography of all resources utilized is provided and will allow trainees with interest to pursue further independent reading. Furthermore, since clinical workload is already heavy, it would be difficult to allot more time to any one capacity-building topic. An exhaustive discussion of all bioethical topics relevant to critical care medicine is not possible nor our intent. However, with the foundational understanding provided, we hope trainees will be able to expand their skills as the law and the field of bioethics evolves. 


\section{Conclusion}

We present in this paper a six module curriculum designed to provide a case-based learning approach that asks trainees to problem-solve and provides them with clinically usable practical points to identify, solve and ideally prevent, commonly encountered bioethical problems in the Intensive Care Unit. This curriculum fulfils a lack of formal bioethics training in critical care, and has been developed to address subject areas desired by trainees for continued learning and deemed necessary in the literature. We hope that our efforts will help make ethical dilemmas less daunting and their management more commonplace.

\section{Compliance with ethical standards}

Conflict of Interest None to declare. Educational Initiatives in Residence Education Grant received from the University of Ottawa Faculty of Medicine post-completion.

\section{References}

Ahern, S.P., T.K. Doyle, F. Marquis, C. Lesk, and Y. Skrobik. 2012. Critically ill patients and end-of-life decision-making: the senior medical resident experience. Advance in Health Science Education 17(1): 121-136.

Crausman, R., and J.D. Armstrong. 1996. Ethically based medical decision making in the intensive care unit residency teaching strategies. Critical Care Clinics 12(1): 71-84.

Downar, J., K. Knickle, J.T. Granton, and L. Hawryluck. 2012. Using standardized family members to teach communication skills and ethical principles to critical care trainees. Critical Care Medicine 40(6): 1814 1819.

Etchells E. 2015. Aid to capacity evaluation Tool. Joint Centre for Bioethics Online resource. Available from: [http://www.jcb.utoronto.ca/tools/ace download.shtml]. Accessed: 3rd March, 2015.

Heaney, M., C. Foot, W.D. Freeman, and J. Fraser. 2007. Ethical issues in withholding and withdrawing lifeprolonging medical treatment in the ICU. Current Anaesthesia and Critical Care 18: 277-283.

Hume D. 1739. A Treatise of Human Nature, Book iii. (1739): Part I, section I, page. 241. Electronic resource. Available from: [http://www.earlymoderntexts.com/pdfs/hume1740book3.pdf]. Accessed: 1st April, 2015.

Huynh, T.N., E.C. Kleerup, J.F. Wiley, et al. 2013. The frequency and cost of treatment perceived to be futile in critical care. Journal of the American Medical Association 173(20): 1887-1894.

Kane, G.C., J. Rowane, and J.E. Fish. 1998. Nationwide perspective on the use of a formal ethics curriculum during critical care fellowship training. Academic Medicine 73(1): 103.

Landry, J., T. Foreman, and M. Kekewich. 2015. Ethical considerations in the regulation of euthanasia and physician-assisted death in Canada. Health Policy. doi:10.1016/j.healthpol.2015.10.002.

Mani RK. 2003. Limitation of life support in the ICU: Ethical issues relating to end-of-life care. International Journal of Critical Care Medicine April-June (7:2), 112-117.

Miles S, Lane L, Bickle J et al. 1989. Medical ethics education: Coming of Age. Academic Medicine 165

Moore, P., I. Kerridge, J. Gillis, S. Jacobe, and D. Isaacs. 2008. Withdrawal of and limitation of life-sustaining treatments in a pediatric intensive care unit and review of the literature. Journal of Pediatrics and Child Health 44: 404-408.

Mularski, R.A., P. Bascom, and M.L. Osborne. 2001. Educational agendas for interdisciplinary end-of-life curricula. Critical Care Medicine 29(2): N16-N23.

Niederman, M.S., and J.T. Berger. 2010. The delivery of futile care is harmful to other patients. Critical Care Medicine 38(10 Supp): S518-S522.

Palda, V.A., K.W. Bowman, R.F. McLean, and M.G. Chapman. 2005. 'Futile' care: Do we provide it? Why? Journal of Critical Care 20: 208. 
Reynolds, S., A.B. Cooper, and M. McKneally. 2005. Withdrawing life-sustaining treatment: ethical considerations. Thoracic Surgery Clinics 15: 469-480.

Royal College of Physicians and Surgeons of Canada. 2005. CanMEDS 2005 Framework. Royal College of Physicians and Surgeons of Canada online resource. Retrieved from: [http://www.royalcollege.ca/portal/ page/portal/rc/canmeds/framework]. Accessed: 14th September, 2014.

Stevens, L., D. Cook, G. Guyatt, L. Griffith, S. Walter, and J. McMullin. 2002. Education, ethics, and end-oflife decisions in the intensive care unit. Critical Care Medicine 30(2): 290-296.

Straus S, and Stelfox T. 2007. Whose life is it anyway? Capacity and Consent in Canada.

Thompson AK, Faith K, Gibson JL, and Upshur REG. 2006. Pandemic influenza preparedness: an ethical framework to guide decision-making. BMC Medical Ethics 7:12, n.p.

Trillium Health. 2015. Ethics at Trillium Health Partners. Online Resource. Available from: [http:// trilliumhealthpartners.ca/aboutus/pages/ethics-at-trillium-health-partners.aspx]. Accessed: 3rd March, 2015.

Van Mook, W., W.S. de Grave, S.L. Gorter, et al. 2009. Fellows' in intensive care medicine views on professionalism and how they learn it. Intensive Care Medicine 36: 300. 